\title{
Is math anxiety caused by a deficit in basic numerical skills? A study using numerical and non-numerical tasks
}

\section{Javier García-Orza \& Patricia Carratalá \\ Universidad de Málaga, Spain \\ jgorza@uma.es}

MATH ANXIETY (MA):

An exaggerated feeling of anxiety when faced with numerical tasks. It is suffered by between 5 and $20 \%$ of people (Ashcraft, 2002).

$\mathrm{MA}$ is negatively correlated with mathematical performance.

\section{THE PROBLEM: WHICH WAS FIRST A DEFICIT IN MATHS OR MATH ANXIETY?}

\section{WORKING MEMORY ACCOUNT}

(Ashcraft $\phi$ cols. 2001, 2005, 2007)

\section{Negative thoughts about math taxes working memory thus} affecting mathematical performance.

EVIDENCE: Only those mathematical tasks complex enough to involve working memory resources (e.g., multidigit operations) are affected by MA (Ashcraft $\$$ Faust, 1994).

\section{BASIC NUMERICAL DEFICIT ACCOUNT}

MA is motivated by low-level deficits in numerical processing that compromise the development of higher level mathematical skills.

EVIDENCE: High MA participants showed a bigger numerical distance effect in an Arabic number comparison task (Maloney et al., 2011). and performed worse in dot enumeration with numbers higher than 4 (Maloney et al., 2011).

IMPORTANT: MOst of these studies did not control for some relevant variables, hence the difficulties found in basic tasks could be motivated by differences in other cognitive processes like speed of processing, working memory, attention... between the high and low math anxiety groups.

Goals: $\quad$ 1. To verify whether a specific deficit in basic mathematical skills is present in high math anxiety people

2. To verify the extent to what these deficits are limited to maths or simply reflect individual differences between groups in several cognitive domains.

Participants

$N$ Males Age Math Anxiety Mood (in months) Score (AMAS)** Anxiety Trait
Anxiety **

Working Memory Score Arithmetic

Low MA group $\begin{array}{llr}15 & 9 & 144.9 \\ 15 & 6 & 145.6\end{array}$ $144.9-14.2$ High MA group 15 $6 \quad 145.6$ 14.2
23.6

30.1 29

19.5 16.3

\section{Experiment 1: Dot comparison}

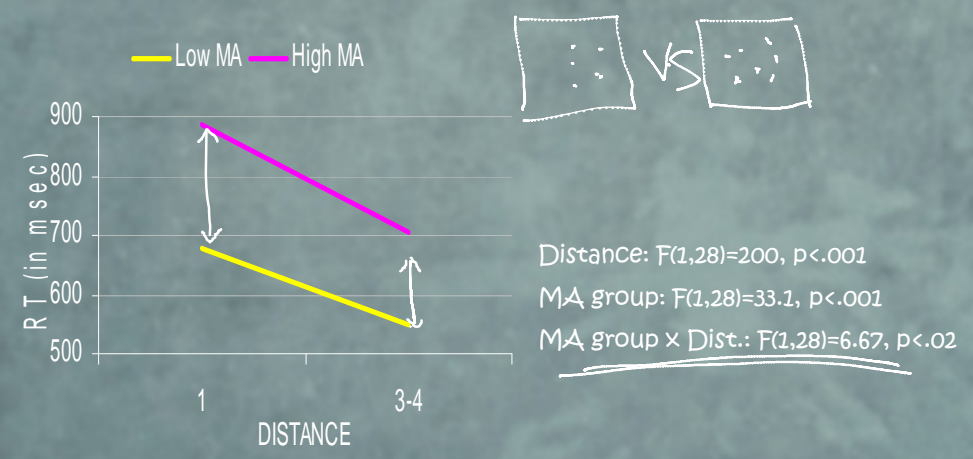

Experiment 3: Physical size comparison

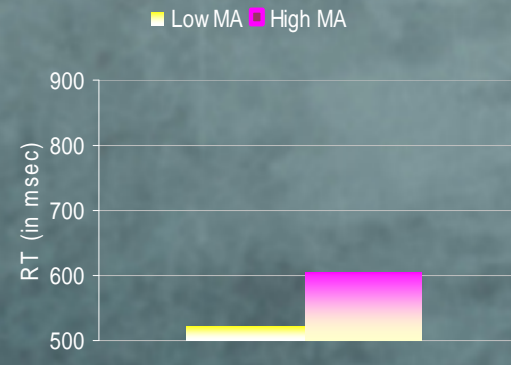

MA group : $t(28)=2.2, p<.04$

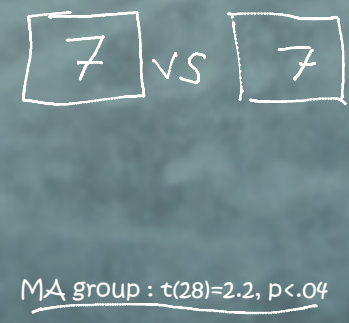

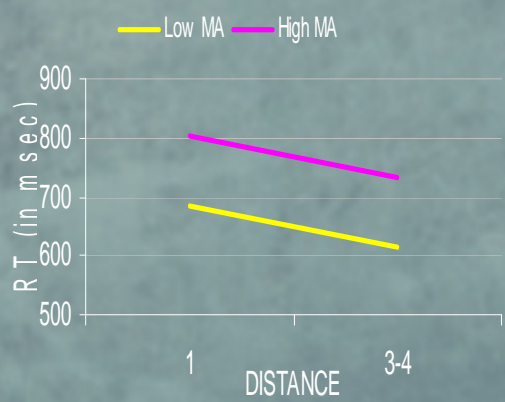

Distance: $F(1,28)=24.4, p<.001$ MA group: $F(1,28)=8.7, p<.001$ MA group $\times$ Dist.: F<1

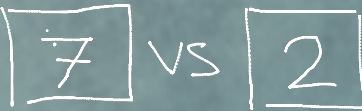

\section{Discussion $\downarrow$ Conclusions}

- Low MA participants showed a more pronounced distance effect in dot comparison task supporting the basic numerical deficit account.

- A similar distance effect, but with differences in response times, was observed in the Arabic comparison task, thus suggesting similar numerical representations from symbolic stimuli in both MA groups. This goes against the basic numerical deficit account.

- Differences in speed were also present in non-numerical tasks (physical size comparison and Posner's matching task).

- Differences between Low and High MA groups also arose regarding trait anxiety but not regarding working memory.

- Caution should be taken before interpreting differences in numerical processing between MA groups.

- The extent to which differences in cognitive processes (e.g., attention, speed of processing...) and general anxiety between MA groups are involved in the differences found in numerical tasks remains to be clarified. 